\title{
Neutrino clustering in the galaxy with a global monopole
}

\author{
Tae Hoon Lef ${ }^{\text {f }}$ and Bruce H. J. McKellar \\ School of Physics, University of Melbourne, Victoria 3010, Australia
}

(Dated: September 3, 2018)

\begin{abstract}
In spherically symmetric, static spacetime, we show that only $j=1 / 2$ fermions can satisfy both Einstein's field equation and Dirac's equation. It is also shown that neutrinos are able to have effective masses and cluster in the galactic halo when they are coupled to a global monopole situated at the galactic core. Astronomical implications of the results are discussed.
\end{abstract}

PACS numbers: 95.30.Sf, 96.40.Tv, 98.35.Gi, $14.80 . \mathrm{Mz}$

\section{INTRODUCTION}

In Barriola and Vilenkin's global monopole solution

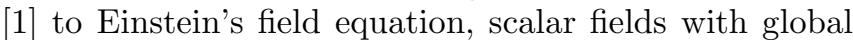
$O(3)$ broken symmetry are minimally coupled to gravity and the background spacetime has deficits of angle. Nucamendi et al. [2] suggested that the global monopole solution could explain the flat rotational velocity curves(FRVC) of stars in galaxies because its energy density is proportional to $1 / r^{2}$ and hence it can be dark matter in the galatic halo. Even if some questions are issued [3] about the global monopole solution for FRVC, various generalized versions of the global monopole were studied as models for dark matter and dark energy [4].

Neutrino clustering was studied by some authors including one of us 50 to explain the continuation of the cosmic ray spectrum beyond the GZK(Greisen, Zatsepen and Kuzmin) cutoff. Neutrino-antineutrino $(\nu-\bar{\nu})$ annihilation to a $Z^{0}$-boson is one possible explanation for the phenomena. But for $\nu-\bar{\nu}$ annihilation into a $Z^{0}$-boson to produce super GZK air shower, a neutrino flux at $E_{\nu} \geq 10^{21} \mathrm{eV}$ with $m_{\nu} \sim$ a few $e V$ and a significant clustering of the relic neutrino density in our galactic halo are required [6].

In this article we investigate if neutrinos can coalesce into neutrino clouds in a curved spacetime. Considering fermions coupled to an $O(3)$ triplet of scalar fields in the most general static metric with spherical symmetry, we show that only the total angular momentum $j=1 / 2$ fermions can satisfy both Einstein's field equation and Dirac's equation. When the global $O(3)$ symmetry of the Lagrangian is spontaneously broken to $U(1)$ at the ground state of the scalar potential in the shape of Mexican hat, we can have a global monopole solution similar to that of Barriola and Vilenkin. Being coupled to a global monopole situated at the galactic center, neutrinos are able to have effective masses and cluster in the galactic halo.

*Permanent address: Department of Physcis, Soongsil University, Seoul 156-743, Korea: thlee@phvsics.ssu.ac.kr

${ }^{\dagger}$ Electronic address: b.mckellar@physics.unimelb.edu.au

\section{GLOBAL $O(3)$ SYMMETRIC MODEL FOR NEUTRINO CLUSTERING}

The action of the global $O(3)$ symmetric model of scalar fields $\Phi^{m}$ and massive neutrinos $\Psi_{n}(m, n=$ $1,2,3)$ minimally coupled to gravity is given by

$$
\begin{aligned}
S & =\int d^{4} x \sqrt{-g} \mathcal{L}, \\
\mathcal{L} & =-\frac{1}{2} g^{\mu \nu} \partial_{\mu} \Phi^{m} \partial_{\nu} \Phi^{m}-V\left(\vec{\Phi}^{2}\right) \\
& +\frac{i}{2}\left(\bar{\Psi}_{n} \gamma^{a} \nabla_{a} \Psi_{n}-\nabla_{a} \bar{\Psi}_{n} \gamma^{a} \Psi_{n}\right)-m_{n} \bar{\Psi}_{n} \Psi_{n} \\
& +\frac{g_{y}}{v} \Phi^{m} \Phi^{m} \bar{\Psi}_{n} \Psi_{n},
\end{aligned}
$$

where $V\left(\vec{\Phi}^{2}\right)$ is a scalar potential and the last term is the scalar-neutrino interaction for which we assume the first leading order term preserving $O(3)$ symmetry, with the symmetry breaking scale $v$ of the more fundamental theory. Varying the action with respect to the fields, we obtain following equations for scalar fields $\Phi^{m}$ and neutrinos $\Psi_{n}$ :

$$
\begin{gathered}
\frac{1}{\sqrt{-g}} \partial_{\mu}\left(\sqrt{-g} g^{\mu \nu} \partial_{\nu} \Phi^{m}\right)-\frac{\partial V}{\partial \Phi^{m}}+2 \frac{g_{y}}{v} \bar{\Psi}_{n} \Psi_{n} \Phi^{m}=0 \\
i \gamma^{a} \nabla_{a} \Psi_{n}-m_{n} \Psi_{n}+\frac{g_{y}}{v} \vec{\Phi}^{2} \Psi_{n}=0
\end{gathered}
$$

where the $\gamma^{a}$-matrices satisfy the Clifford algebra in a locally flat inertial coordinate;

$$
\left\{\gamma^{a}, \gamma^{b}\right\}=-2 \eta^{a b}
$$

with

$$
\eta^{a b}=\operatorname{Diag}(-1,1,1,1),
$$

and the covariant derivative

$$
\nabla_{a}=e^{\mu}{ }_{a}\left(\partial_{\mu}+\Gamma_{\mu}\right)
$$

is constructed from the vierbein $e^{\mu}{ }_{a}$ and spin connection $\Gamma_{\mu}$, which we give explicitly in Eqs. (25)-(31).

Using the standard definition of the energy-momentum tensor

$$
T_{\mu \nu} \equiv-\frac{2}{\sqrt{-g}} \frac{\delta S}{\delta g^{\mu \nu}}=-\frac{e_{a \mu}}{\operatorname{det}\{e\}} \frac{\delta S}{\delta e_{a}^{\nu}}
$$


we have

$$
\begin{aligned}
T_{\mu \nu} & =\partial_{\mu} \Phi^{m} \partial_{\nu} \Phi^{m}-g_{\mu \nu}\left[\frac{1}{2} \partial^{\beta} \Phi^{m} \partial_{\beta} \Phi^{m}+V\left(\vec{\Phi}^{2}\right)\right] \\
& -\frac{i}{4}\left[\left(\bar{\Psi}_{n} \gamma_{\mu} \nabla_{\nu} \psi_{n}-\nabla_{\nu} \bar{\Psi}_{n} \gamma_{\mu} \Psi_{n}\right)+(\mu \leftrightarrow \nu)\right]
\end{aligned}
$$

The energy-momentum tensor allows us to construct the Einstein equation,

$$
G_{\mu \nu}=\kappa T_{\mu \nu}
$$

where $G_{\mu \nu}$ is the Einstein tensor

$$
G_{\mu \nu}=R_{\mu \nu}-\frac{1}{2} g_{\mu \nu} R
$$

with Ricci tensor $R_{\mu \nu}$ and $\kappa=8 \pi G$. fields

When we consider the following potential of scalar

$$
V\left(\vec{\Phi}^{2}\right)=\frac{\lambda}{4}\left(\vec{\Phi}^{2}-\eta^{2}\right)^{2}
$$

with a constant $\eta$, the global $O(3)$ symmetry is spontaneously broken to $U(1)$. The scalar field configuration describing a global monopole is known to be given by the hedgehog ansatz,

$$
\Phi^{m}=F(r) \frac{x_{m}}{r}
$$

with a real function $F(r)$ and $r=\left(x_{m} x_{m}\right)^{1 / 2}=\left(x^{2}+\right.$ $\left.y^{2}+z^{2}\right)^{1 / 2}$,

Assume the line element of the spherically symmetric, static spacetime as

$$
d s^{2}=-\delta^{2}(r) \alpha^{2}(r) d t^{2}+\frac{d r^{2}}{\alpha^{2}(r)}+r^{2} d \theta^{2}+r^{2} \sin ^{2} \theta d \phi^{2}
$$

In the Cartesian coordinates,

$$
x^{\mu}=\left(t, x^{m}\right)=(t, x, y, z), \quad \text { for } m=1,2,3,
$$

the line element can be written by

$$
d s^{2}=g_{\mu \nu} d x^{\mu} d x^{\nu}
$$

with the metric tensor

$$
g_{\mu \nu}=-\delta^{2} \alpha^{2} \delta_{\mu}^{t} \delta^{t}{ }_{\nu}+\left[\delta_{m n}+\left(\frac{1}{\alpha^{2}}-1\right) \frac{x_{m} x_{n}}{r^{2}}\right] \delta^{m}{ }_{\mu} \delta^{n}{ }_{\nu}
$$

and its inverse

$$
g^{\mu \nu}=-\frac{1}{\delta^{2} \alpha^{2}} \delta^{\mu}{ }_{t} \delta^{\nu}{ }_{t}+\left[\delta_{m n}+\left(\alpha^{2}-1\right) \frac{x_{m} x_{n}}{r^{2}}\right] \delta^{\mu}{ }_{m} \delta^{\nu}{ }_{n} .
$$

From the standard definition of Christoffel symbols

$$
\Gamma_{\alpha \beta}^{\mu}=\frac{1}{2} g^{\mu \nu}\left(g_{\nu \alpha, \beta}+g_{\nu \beta, \alpha}-g_{\alpha \beta, \nu}\right),
$$

we derive

$$
\begin{aligned}
\Gamma_{t m}^{t} & =\frac{x_{m}}{2 r}\left(\frac{\delta^{2},{ }_{r}}{\delta^{2}}+\frac{\alpha^{2},{ }_{r}}{\alpha^{2}}\right), \\
\Gamma_{t t}^{l} & =\frac{x_{l} \alpha^{2}}{2 r}\left(\delta^{2} \alpha^{2},{ }_{r}+\delta^{2},{ }_{r} \alpha^{2}\right), \\
\Gamma_{m n}^{l} & =\frac{x_{l} \alpha^{2}}{2 r}\left[-\frac{\alpha^{2},{ }_{r}}{\alpha^{4}} \frac{x_{m} x_{n}}{r^{2}}+\left(\alpha^{-2}-1\right)\left(\delta_{m n}-\frac{x_{m} x_{n}}{r^{2}}\right) \frac{2}{r}\right], \\
\text { Others } & =0 .
\end{aligned}
$$

In the metric given in Eq. (14), scalar field equation reads

$$
\begin{aligned}
& \alpha^{2} F,_{r r}+\frac{\alpha^{2}}{2} F,_{r}\left(\frac{\delta^{2},_{r}}{\delta^{2}}+2 \frac{\alpha^{2},_{r}}{\alpha^{2}}+\frac{4}{r}\right)-\frac{2 F}{r^{2}} \\
& -\frac{\partial V}{\partial F}+\frac{2 g_{y}}{v} \bar{\Psi}_{n} \Psi_{n} F=0
\end{aligned}
$$

where $F,_{r} \equiv \frac{\partial F}{\partial r}, F,_{r r} \equiv \frac{\partial^{2} F}{\partial r^{2}}, \ldots$.

We rewrite the line element as

$$
d s^{2}=\eta_{a b} e^{a} e^{b}
$$

with inverse of $\eta^{a b}, \eta_{a b}=\operatorname{Diag}(-1,1,1,1)$ and

$$
e^{0}=\alpha \delta d t, e^{1}=\frac{d r}{\alpha}, e^{2}=r d \theta, e^{3}=r \sin \theta d \phi .
$$

Putting

$$
e^{a}=e^{a}{ }_{\mu} d x^{\mu}
$$

we find the vierbein given by

$$
e_{\mu}^{a}=\left(\begin{array}{cccc}
\alpha \delta & 0 & 0 & 0 \\
0 & \frac{1}{\alpha} \sin \theta \cos \phi & \frac{1}{\alpha} \sin \theta \sin \phi & \frac{1}{\alpha} \cos \theta \\
0 & \cos \theta \cos \phi & \cos \theta \sin \phi & -\sin \theta \\
0 & -\sin \phi & \cos \phi & 0
\end{array}\right)
$$

and their inverses

$$
\begin{aligned}
e_{b}^{\nu} & =\left(\begin{array}{cccc}
\frac{1}{\alpha \delta} & 0 & 0 & 0 \\
0 & \alpha \sin \theta \cos \phi & \cos \theta \cos \phi & -\sin \phi \\
0 & \alpha \sin \theta \sin \phi & \cos \theta \sin \phi & \cos \phi \\
0 & \alpha \cos \theta & -\sin \theta & 0
\end{array}\right) \\
& =\left(\begin{array}{cccc}
\frac{1}{\alpha \delta} & 0 & 0 & 0 \\
0 & \left(\alpha \frac{\partial x^{m}}{\partial r}\right) & \left(\frac{1}{r} \frac{\partial x^{m}}{\partial \theta}\right) & \left(\frac{1}{r \sin \theta} \frac{\partial x^{m}}{\partial \phi}\right)
\end{array}\right) .
\end{aligned}
$$

The vierbein satisfy the relations

$$
e^{a}{ }_{\mu} e^{\mu}{ }_{b}=\delta_{b}^{a}
$$

$$
e^{\nu}{ }_{a} e_{\mu}^{a}=\delta_{\mu}^{\nu}
$$

The spin connection which appeared in Eq. (7) is defined as

$$
\Gamma_{\mu}=-\frac{1}{4} \gamma^{a} \gamma^{b} e_{\nu a} e_{b ; \mu}^{\nu}
$$


with

$$
e_{b ; \alpha}^{\nu}=e_{b, \alpha}^{\nu}+\Gamma_{\alpha \beta}^{\nu} e_{b}^{\beta} .
$$

We calculate the spin connections from the above Eq. (20) and Eq. (30), and obtain

$$
\begin{aligned}
\Gamma_{t} & =\frac{1}{4} \gamma^{0} \gamma^{1}\left(2 \alpha^{2} \delta,_{r}+\delta \alpha^{2},_{r}\right) \\
\Gamma_{x} & =\frac{1}{2} \gamma^{1} \gamma^{2} \frac{\alpha \cos \theta \cos \phi}{r}-\frac{1}{2} \gamma^{2} \gamma^{3} \frac{\cos \theta \sin \phi}{r \sin \theta} \\
& +\frac{1}{2} \gamma^{3} \gamma^{1} \frac{\alpha \sin \phi}{r}, \\
\Gamma_{y} & =\frac{1}{2} \gamma^{1} \gamma^{2} \frac{\alpha \cos \theta \sin \phi}{r}+\frac{1}{2} \gamma^{2} \gamma^{3} \frac{\cos \theta \cos \phi}{r \sin \theta} \\
& -\frac{1}{2} \gamma^{3} \gamma^{1} \frac{\alpha \cos \phi}{r}, \\
\Gamma_{z} & =-\frac{1}{2} \gamma^{1} \gamma^{2} \frac{\alpha \sin \theta}{r},
\end{aligned}
$$

which satisfy the Cartan structure equation,

$$
\left[\gamma^{\nu}, \Gamma_{\mu}\right]=\gamma_{; \mu}^{\nu}
$$

\section{SPHERICAL SYMMETRY AND $j=\frac{1}{2}$ FERMIONS}

We can show that neutrino field equations given by

$$
i \gamma^{a} e^{\mu}{ }_{a}\left(\partial_{\mu}+\Gamma_{\mu}\right) \Psi_{n}-m_{n} \Psi_{n}+\frac{g_{y}}{v} \vec{\Phi}^{2} \Psi_{n}=0
$$

become

$$
\begin{aligned}
& \gamma^{0} \frac{i}{\alpha \delta} \partial_{t} \Psi_{n}+\gamma^{1} i \alpha \hat{D}_{r} \Psi_{n}+\gamma^{2} \frac{i}{r}\left(\partial_{\theta}+\frac{\cos \theta}{2 \sin \theta}\right) \Psi_{n} \\
& +\gamma^{3} \frac{i}{r \sin \theta} \partial_{\phi} \Psi_{n}-\left(m_{n}-\frac{g_{y}}{v} \vec{\Phi}^{2}\right) \Psi_{n}=0
\end{aligned}
$$

in the spherically symmetric and static metric in Eq. (14). Equivalently, we can write the last equation as

$$
i \partial_{t} \Psi_{n}=\hat{H} \Psi_{n}
$$

where the Hamiltonian is defined as

$$
\hat{H}=\alpha \delta\left[\gamma^{0} \gamma^{1} \frac{\alpha}{i} \hat{D}_{r}+\gamma^{1} \frac{1}{i r} \hat{k}+\gamma^{0}\left(m_{n}-\frac{g_{y}}{v} \vec{\Phi}^{2}\right)\right],
$$

with

$$
\hat{D}_{r}=\partial_{r}+\frac{1}{r}+\frac{1}{4}\left(\frac{\delta^{2},_{r}}{\delta^{2}}+\frac{\alpha^{2},_{r}}{\alpha^{2}}\right) .
$$

Here we have defined the operator $\hat{k}$ as $[8$

$$
\hat{k} \equiv i \gamma^{0} \gamma^{1}\left(\gamma^{2} \frac{1}{i \sqrt{\sin \theta}} \partial_{\theta} \sqrt{\sin \theta}+\gamma^{3} \frac{1}{i \sin \theta} \partial_{\phi}\right),
$$

which commutes with the Hamiltonian operator $\hat{H}$

$$
[\hat{k}, \hat{H}]=0 .
$$

Taking the representation of $\gamma$-matrices to be direct products of independnet Pauli matrices $\sigma_{i}, \rho_{j}(\vec{\rho} \otimes \vec{\sigma}$ representation) [7] [8],

$$
\gamma^{0}=\rho_{2}, \gamma^{1}=i \rho_{1}, \gamma^{2}=-i \rho_{3} \sigma_{3}, \gamma^{3}=-i \rho_{3} \sigma_{1},
$$

we can represent $\hat{k}$ in 2 -dimensional $\sigma$-space

$$
\hat{k}=\sigma_{3} \frac{1}{i \sqrt{\sin \theta}} \partial_{\theta} \sqrt{\sin \theta}+\sigma_{1} \frac{1}{i \sin \theta} \partial_{\phi},
$$

and we can solve the eigenvalue equation,

$$
\hat{k} \mathcal{Y}_{k}^{m}(\theta, \phi)=k \mathcal{Y}_{k}^{m}(\theta, \phi),
$$

with 2-dimensional spinor spherical harmonics 8

$$
\begin{aligned}
& \mathcal{Y}_{k}^{m}(\theta, \phi)=\frac{e^{i m \phi}}{\sqrt{2 \pi}}\left[\frac{(j+m) !}{(j-m) !}\right]^{1 / 2} \frac{\left(\tan \frac{\theta}{2}\right)^{\sigma_{2} / 2}}{\sin ^{m} \theta} \times \\
& \left(\frac{\partial}{\partial \cos \theta}\right)^{j-m} \frac{\sin ^{2 j} \theta}{2^{j}\left(j-\frac{1}{2}\right) !}\left(\tan \frac{\theta}{2}\right)^{-\sigma_{2}} u_{k},
\end{aligned}
$$

where

$$
u_{k}=\frac{1}{\sqrt{2}|k|}\left(\begin{array}{c}
|k| \\
k
\end{array}\right)
$$

with $k= \pm\left(j+\frac{1}{2}\right)$ and $j=l \pm \frac{1}{2}$. In the representation (41) for $\gamma$-matrices, the Hamiltonian is expressed in terms of 2-dimensional $\rho$-matrices

$$
\hat{H}=\alpha \delta\left[\rho_{3} \frac{\alpha}{i} \hat{D}_{r}+\rho_{1} \frac{1}{r} \hat{k}+\rho_{2}\left(m_{n}-\frac{g_{y}}{v} \vec{\Phi}^{2}\right)\right]
$$

The eigenfunctions of both $\hat{H}$ and $\hat{k}$ can be written as direct products of the $\rho$-space spinor $\psi_{n}^{(k m)}$ and $\sigma$-space spinor $\mathcal{Y}_{k}^{m}$,

$$
\Psi_{n}(t, r, \theta, \phi)=e^{-i E_{n} t} \sum_{-j \leq m \leq j} \psi_{n}^{(k m)}(r) \mathcal{Y}_{k}^{m}(\theta, \phi),
$$

and the neutrino equations $i \partial_{t} \Psi_{n}=\hat{H} \Psi_{n}$ then read

$$
\frac{E_{n}}{\alpha \delta} \psi_{n}^{(k m)}=\rho_{3} \frac{\alpha}{i} \hat{D}_{r} \psi_{n}^{(k m)}+\left[\rho_{1} \frac{k}{r}+\rho_{2}\left(m_{n}-\frac{g_{y}}{v} \vec{\Phi}^{2}\right)\right] \psi_{n}^{(k m)} .
$$

Moreover, if we put

$$
\psi_{n}^{(k m)}(r) \equiv \frac{1}{r}\left(\alpha^{2} \delta^{2}\right)^{\frac{-1}{4}} R_{n}^{(k m)}(r),
$$

since

$$
\hat{D}_{r} \psi_{n}^{(k m)}=\frac{1}{r}\left(\alpha^{2} \delta^{2}\right)^{\frac{-1}{4}} \partial_{r} R_{n}^{(k m)}
$$

then we get

$$
\frac{E_{n}}{\alpha \delta} R_{n}^{(k m)}=\rho_{3} \frac{\alpha}{i} \partial_{r} R_{n}^{(k m)}+\left[\rho_{1} \frac{k}{r}+\rho_{2}\left(m_{n}-\frac{g_{y}}{v} \vec{\Phi}^{2}\right)\right] R_{n}^{(k m)} .
$$


Setting

$$
R_{n}^{(k m)}=\left(\begin{array}{c}
\varphi_{n}^{(k m)} \\
\chi_{n}^{(k m)}
\end{array}\right),
$$

we obtain the coupled equations,

$$
\begin{aligned}
\left(\frac{E_{n}}{\alpha \delta}+i \alpha \partial_{r}\right) \varphi_{n}^{(k m)} & =\left[\frac{k}{r}-i\left(m_{n}-\frac{g_{y}}{v} \vec{\Phi}^{2}\right)\right] \chi_{n}^{(k m)}(53) \\
\left(\frac{E_{n}}{\alpha \delta}-i \alpha \partial_{r}\right) \chi_{n}^{(k m)} & =\left[\frac{k}{r}+i\left(m_{n}-\frac{g_{y}}{v} \vec{\Phi}^{2}\right] \varphi_{n}^{(k m)},\right.
\end{aligned}
$$

asymptotic solutions to which are given in the following section.

Einstein's equation $G_{\mu \nu}=\kappa T_{\mu \nu}$ can be rewriten as

$$
R_{\mu \nu}=\kappa\left(T_{\mu \nu}-\frac{1}{2} g_{\mu \nu} T_{\alpha}^{\alpha}\right) .
$$

Substituting Eq. (9) into the Einstein's equation in Eq. (54) gives

$$
\begin{aligned}
R_{\mu \nu}= & \kappa\left[\partial_{\mu} \Phi^{m} \partial_{\nu} \Phi^{m}+g_{\mu \nu} V+\frac{i}{4} g_{\mu \nu} \Sigma\right. \\
& -\frac{i}{4}\left(\bar{\Psi}_{n} \gamma_{\mu} \nabla_{\nu} \Psi_{n}-\nabla_{\mu} \bar{\Psi}_{n} \gamma_{\nu} \Psi_{n}\right. \\
& \left.\left.+\bar{\Psi}_{n} \gamma_{\nu} \nabla_{\mu} \Psi_{n}-\nabla_{\nu} \bar{\Psi}_{n} \gamma_{\mu} \Psi_{n}\right)\right],
\end{aligned}
$$

with

$$
\Sigma=e_{c}^{\alpha}\left(\bar{\Psi}_{n} \gamma^{c} \partial_{\alpha} \Psi_{n}-\partial_{\alpha} \bar{\Psi}_{n} \gamma^{c} \Psi_{n}\right) .
$$

Elements of Ricci tensor calculated from metric coefficients in Eq. (14) and the ansatz for $\Phi^{m}$ in Eq. (13) are substituted into Eq. (55), to give the set of equations

$$
\begin{aligned}
& \frac{1}{2 \delta^{2}}\left(\delta^{2} \alpha^{2}\right){ }_{r r}-\frac{\left(\delta^{2} \alpha^{2}\right),_{r}}{4 \delta^{2}} \frac{\delta^{2},_{r}}{\delta^{2}}+\frac{1}{r} \frac{\left(\delta^{2} \alpha^{2}\right),_{r}}{\delta^{2}}= \\
& \kappa\left[-V-\frac{i}{4} \Sigma+\frac{i}{2 \delta \alpha}\left(\bar{\Psi}_{n} \gamma^{0} \partial_{t} \Psi_{n}-\partial_{t} \bar{\Psi}_{n} \gamma^{0} \Psi_{n}\right)\right], \\
& -\frac{1}{2 \delta^{2}}\left(\delta^{2} \alpha^{2}\right){ }_{r r}+\frac{\left(\delta^{2} \alpha^{2}\right){ }_{r}}{4 \delta^{2}} \frac{\delta^{2},_{r}}{\delta^{2}}-\frac{1}{r} \alpha^{2},_{r}= \\
& \kappa\left[\alpha^{2}\left(F,{ }_{r}\right)^{2}+V+\frac{i}{4} \Sigma\right. \\
& \left.-\frac{i \alpha}{2}\left(\bar{\Psi}_{n} \gamma^{1} \partial_{r} \Psi_{n}-\partial_{r} \bar{\Psi}_{n} \gamma^{1} \Psi_{n}\right)\right], \\
& \frac{1}{r^{2}}\left(1-\alpha^{2}\right)-\frac{1}{r} \alpha^{2},_{r}-\frac{\alpha^{2}}{2 r} \frac{\delta^{2},_{r}}{\delta^{2}}= \\
& \kappa\left[\frac{F^{2}}{r^{2}}+V+\frac{i}{4} \Sigma\right. \\
& \left.-\frac{i}{2 r}\left(\bar{\Psi}_{n} \gamma^{2} \partial_{\theta} \Psi_{n}-\partial_{\theta} \bar{\Psi}_{n} \gamma^{2} \Psi_{n}\right)\right], \\
& \frac{1}{r^{2}}\left(1-\alpha^{2}\right)-\frac{1}{r} \alpha^{2}{ }_{r}-\frac{\alpha^{2}}{2 r} \frac{\delta^{2},_{r}}{\delta^{2}}= \\
& \kappa\left[\frac{F^{2}}{r^{2}}+V+\frac{i}{4} \Sigma\right. \\
& \left.-\frac{i}{2 r \sin \theta}\left(\bar{\Psi}_{n} \gamma^{3} \partial_{\phi} \Psi_{n}-\partial_{\phi} \bar{\Psi}_{n} \gamma^{3} \Psi_{n}\right)\right] .
\end{aligned}
$$

The first in above equations is the $(t, t)$-component of Eq. (55), the second $(r, r)$-component, the third $(\theta, \theta)$ component, and the last $(\phi, \phi)$-component, respectively.

$(\theta, \theta)$-component of the Einstein equation is same as $(\phi, \phi)$-component of Einstein equation if

$\bar{\Psi}_{n} \gamma^{2} \partial_{\theta} \Psi_{n}-\partial_{\theta} \bar{\Psi}_{n} \gamma^{2} \Psi_{n}=\frac{1}{\sin \theta}\left(\bar{\Psi}_{n} \gamma^{3} \partial_{\phi} \Psi_{n}-\partial_{\phi} \bar{\Psi}_{n} \gamma^{3} \Psi_{n}\right)$

Using $\vec{\rho} \otimes \vec{\sigma}$ representation for $\gamma$-matrices and the mathematical identities;

$$
\begin{aligned}
\sigma_{3} \partial_{\theta} \mathcal{Y}_{k}^{m}=\quad & \left( \pm \sigma_{3} m \cot \theta \pm \sigma_{1} \frac{i}{2 \sin \theta}\right) \mathcal{Y}_{k}^{m} \\
& \pm \sigma_{3} e^{\mp i \phi} \sqrt{(j \pm m+1)(j \mp m)} \mathcal{Y}_{k}^{m \pm 1}
\end{aligned}
$$

this condition becomes

$$
\begin{aligned}
& \sum_{m, m^{\prime}} \psi_{n}^{\dagger\left(k m^{\prime}\right)} \rho_{1} \psi_{n}^{(k m)} \times \\
& {\left[\mathcal{Y}_{k}^{\dagger m^{\prime}}\left\{ \pm \sigma_{3}\left(m-m^{\prime}\right) \cot \theta \pm \sigma_{1} \frac{i}{\sin \theta}\right\} \mathcal{Y}_{k}^{m}\right.} \\
& \pm \mathcal{Y}_{k}^{\dagger m^{\prime}} \sigma_{3} e^{\mp i \phi} \sqrt{(j \pm m+1)(j \mp m)} \mathcal{Y}_{k}^{m \pm 1} \\
& \left.\mp \mathcal{Y}_{k}^{\dagger m^{\prime} \pm 1} \sigma_{3} e^{ \pm i \phi} \sqrt{\left(j \pm m^{\prime}+1\right)\left(j \mp m^{\prime}\right)} \mathcal{Y}_{k}^{m}\right] \\
& =\sum_{m, m^{\prime}} \psi_{n}^{\dagger\left(k m^{\prime}\right)} \rho_{1} \psi_{n}^{(k m)}\left(m+m^{\prime}\right) \mathcal{Y}_{k}^{\dagger m^{\prime}} \sigma_{1} \frac{i}{\sin \theta} \mathcal{Y}_{k}^{m} .
\end{aligned}
$$

The condition (63) can be satisfied if

$$
\begin{aligned}
m & =m^{\prime}, \\
m+m^{\prime} & =2 m= \pm 1, \\
j & =\frac{1}{2},
\end{aligned}
$$

since

$$
\mathcal{Y}_{k\left(j=\frac{1}{2}\right)}^{ \pm \frac{1}{2} \pm 1}=0 \text {. }
$$

Moreover, explicit calculations show us that

$$
\begin{aligned}
\left(\mathcal{Y}_{k\left(j=\frac{1}{2}\right)}^{ \pm \frac{1}{2}}\right)^{\dagger} \mathcal{Y}_{k\left(j=\frac{1}{2}\right)}^{ \pm \frac{1}{2}} & =\frac{1}{4 \pi} \\
\left(\mathcal{Y}_{k\left(j=\frac{1}{2}\right)}^{ \pm \frac{1}{2}}\right)^{\dagger} \sigma_{1} \mathcal{Y}_{k\left(j=\frac{1}{2}\right)}^{ \pm \frac{1}{2}} & = \pm \frac{\sin \theta}{4 \pi} \frac{k}{|k|}
\end{aligned}
$$

These make Eqs. (57)-(60) $\theta$ - and $\phi$-independent, which is consistent with spherically symmetric metric in Eq. (14), when we consider only $\left(j=\frac{1}{2}, m=\frac{1}{2}\right)$ or $(j=$ $\left.\frac{1}{2}, m=-\frac{1}{2}\right)$ fermions.

For the case $\left(j=\frac{1}{2}, m=\frac{1}{2}\right)$ or $\left(j=\frac{1}{2}, m=-\frac{1}{2}\right)$ with $k= \pm 1$, neutrino wave function is

$$
\Psi_{n}(t, r, \theta, \phi)=e^{-i E_{n} t} \psi_{n}^{\left(k, \pm \frac{1}{2}\right)}(r) \mathcal{Y}_{k}^{ \pm \frac{1}{2}}(\theta, \phi) .
$$

Let $\psi_{n}(r) \equiv \psi_{n}^{\left(k, \frac{1}{2}\right)}(r)$ or $\psi_{n}^{\left(k,-\frac{1}{2}\right)}(r)$ and

$$
\psi_{n}(r)=\frac{1}{r}\left(\alpha^{2} \delta^{2}\right)^{-\frac{1}{4}}\left(\begin{array}{c}
\varphi_{n} \\
\chi_{n}
\end{array}\right) .
$$


In this case the term $\Sigma$ in Eq. (56) reads

$$
\Sigma=-\frac{i}{r^{2} \alpha^{2} \delta^{2}} q_{0}+\frac{i}{r^{2} \delta} q_{1}+\frac{i}{r^{2} \alpha \delta} q_{k},
$$

with

$$
\begin{gathered}
q_{0}=\frac{2 E_{n}}{4 \pi} r^{2} \alpha \delta \psi_{n}^{\dagger} \psi_{n}=\frac{2 E_{n}}{4 \pi}\left(\varphi_{n}^{*} \varphi_{n}+\chi_{n}^{*} \chi_{n}\right), \\
q_{1}=\frac{-i}{4 \pi} r^{2} \alpha \delta\left(\psi_{n}^{\dagger} \rho_{3} \partial_{r} \psi_{n}-\partial_{r} \psi_{n}^{\dagger} \rho_{3} \psi_{n}\right) \\
=\frac{-i}{4 \pi}\left(\varphi_{n}^{*} \partial_{r} \varphi_{n}-\partial_{r} \varphi_{n}^{*} \varphi_{n}\right. \\
\left.-\chi_{n}^{*} \partial_{r} \chi_{n}+\partial_{r} \chi_{n}^{*} \chi_{n}\right), \\
q_{k}=\frac{1}{2 \pi} r \alpha \delta \frac{k}{|k|} \psi_{n}^{\dagger} \rho_{1} \psi_{n}=\frac{1}{2 \pi r} \frac{k}{|k|}\left(\chi_{n}^{*} \varphi+\varphi_{n}^{*} \chi_{n}\right), \\
q_{2}=\frac{1}{4 \pi} r^{2} \alpha \delta \psi_{n}^{\dagger} \rho_{2} \psi_{n}=\frac{i}{4 \pi}\left(\chi_{n}^{*} \varphi-\varphi_{n}^{*} \chi_{n}\right),
\end{gathered}
$$

Using the last equations Einstein's equations are given by

$$
\begin{gathered}
\frac{1}{2 \delta^{2}}\left(\delta^{2} \alpha^{2}\right),_{r r}-\frac{\left(\delta^{2} \alpha^{2}\right),_{r}}{4 \delta^{2}} \frac{\delta^{2},_{r}}{\delta^{2}}+\frac{1}{r} \frac{\left(\delta^{2} \alpha^{2}\right),_{r}}{\delta^{2}} \\
=\kappa\left[-V+\frac{1}{4 r^{2}}\left(\frac{q_{0}}{\delta^{2} \alpha^{2}}+\frac{q_{1}}{\delta}+\frac{q_{k}}{\alpha \delta}\right)\right], \\
\frac{\alpha^{2}}{r} \frac{\delta^{2},_{r}}{\delta^{2}}=\kappa\left[\alpha^{2}\left(F,{ }_{r}\right)^{2}+\frac{1}{2 r^{2}}\left(\frac{q_{0}}{\delta^{2} \alpha^{2}}+\frac{q_{1}}{\delta}\right)\right], \\
\frac{1}{r^{2}}\left(1-\alpha^{2}\right)-\frac{1}{r} \alpha^{2},{ }_{r}-\frac{\alpha^{2}}{2 r} \frac{\delta^{2},_{r}}{\delta^{2}}=\kappa\left[\frac{F^{2}}{r^{2}}+V+\frac{1}{4 r^{2}}\left(\frac{q_{0}}{\delta^{2} \alpha^{2}}-\frac{q_{1}}{\delta}\right)\right],
\end{gathered}
$$

and scalar field equation

$$
\begin{aligned}
& \alpha^{2} F,_{r r}+\frac{\alpha^{2}}{2} F,_{r}\left(\frac{\delta^{2},_{r}}{\delta^{2}}+2 \frac{\alpha^{2},_{r}}{\alpha^{2}}+\frac{4}{r}\right)-\frac{2 F}{r^{2}} \\
& -\frac{\partial V}{\partial F}+\frac{2 g_{y}}{v} \frac{q_{2}}{r^{2} \delta \alpha} F=0 .
\end{aligned}
$$

\section{ASYMPTOTIC SOLUTIONS FOR LARGE $r$}

As the global monopole solution [1], one component $\alpha(r)$ of the metric in Eq. (14), far away from the galactic core, can be asymptotically taken as

$$
\alpha^{2} \simeq \alpha_{o}^{2} \equiv c-\frac{2 M}{r}
$$

with a constant $c$ when

$$
F \simeq \eta, \quad V\left(\eta^{2}\right) \simeq 0, \quad \frac{\partial V}{\partial F}\left(\eta^{2}\right) \simeq 0 .
$$

Analysing Eqs. (74)-(77) with the assumption that $q_{i}$ 's in Eqs. (70)-(73) are $\mathcal{O}\left(r^{0}\right)$, we can get an asymptotic solution for another component of the metric in Eq. (14) as

$$
\delta^{2} \simeq A^{2} \ln \left(\frac{r}{2 M}\right)
$$

with a constant $A$. In this limit with the help of Eq. (68) the neutrino equation (53) becomes

$$
\partial_{r}^{2} \varphi_{n} \simeq-q^{2}(r) \varphi_{n}
$$

with

$$
q(r) \equiv \sqrt{\frac{E_{n}^{2}}{\delta^{2} \alpha_{o}^{4}}-\frac{m_{o . n}^{2}}{\alpha_{o}{ }^{2}}}
$$

and $m_{o . n}=m_{n}-\frac{g_{y}}{v} \eta^{2}$.

In the region

$$
0<\ln \left(\frac{r}{2 M}\right) \leq \frac{E_{n}{ }^{2}}{A^{2} \alpha_{o}{ }^{2} m^{2}{ }_{o . n}}
$$

we have an asymptotic solution to Eq. (81),

$$
\begin{aligned}
\varphi_{n} & \simeq A_{n} e^{i q(r) r}+B_{n} e^{-i q(r) r}, \\
\chi_{n} & \simeq \frac{i}{m_{o . n}}\left[A_{n}\left(\frac{E_{n}}{\alpha_{o} \delta}-\alpha_{o} q(r)\right) e^{+i q(r) r}\right. \\
& \left.+B_{n}\left(\frac{E_{n}}{\alpha_{o} \delta}+\alpha_{o} q(r)\right) e^{-i q(r) r}\right] .
\end{aligned}
$$

With above asymptotic solutions to Dirac's equation for neutrinos, the $q_{i}$ in Eqs. (70)-(73) are given by

$$
\begin{aligned}
q_{0} & \simeq \frac{E_{n}}{\pi}\left[B_{n}^{2}\left(1+\frac{1}{m^{2}{ }_{o . n}}\left(\frac{E_{n}{ }^{2}}{\alpha_{o}^{2} \delta^{2}}+\alpha_{o}^{2} q^{2}(r)\right)\right)\right. \\
& \left.+A_{n} B_{n}\left(1+m^{2}{ }_{o . n}\right) \cos (2 q r)\right], \\
q_{1} & \simeq \frac{2 E_{n}}{\pi} B_{n}^{2} \frac{q^{2}}{\delta}, \\
q_{k} & \simeq \frac{2 k}{\pi r|k|} A_{n} B_{n} \frac{\alpha_{o} q}{m_{o . n}} \sin (2 q r), \\
q_{2} & \simeq \frac{E_{n}}{\pi m_{o . n} \alpha_{o} \delta}\left[A_{n} B_{n} \cos (2 q r)+B_{n}^{2}\right],
\end{aligned}
$$

with real constants $A_{n}$ and $B_{n}$ such that $\left|A_{n}\right|=\left|B_{n}\right|$. The $q_{i}$ 's are $\mathcal{O}\left(r^{0}\right)$ or less and so are consistent with the assumption of Eq. (80). For $r>r_{o}$ with $\ln \left(\frac{r_{o}}{2 M}\right) \equiv$ $\frac{E_{n}{ }^{2}}{A^{2} \alpha_{o}^{2} m^{2}{ }^{2}, n}, q(r)$ in Eq. (82) is pure imaginary and then wave functions of neutrinos become to be multiplied by exponentially decaying factors. Therefore we get neutrino clustering with the radius $r_{o}$. Taking $r_{o} \simeq 10^{23} \mathrm{~cm}$ which corresponds to the radius of our Galaxy and assuming that there exists a supermassive black hole at the center of Galaxy with a mass $M_{S B H} \simeq 3 \times 10^{6} M_{\odot}$ [12], we can estimate the energy of neutrinos at $E_{n} \simeq$ $5 m_{o . n}$, where we have used the relations, $\frac{E_{n}{ }^{2}}{A^{2} \alpha_{o}^{2} m^{2}{ }^{2} . n} \simeq$ $\ln \left(\frac{r_{o}}{2 M_{S B H}}\right)$ and $A^{2} \alpha_{o}^{2} \simeq \mathcal{O}(1)$. 


\section{ASYMPTOTIC SOLUTIONS FOR SMALL $r$}

Next let us study the small $r$ behaviors of components of the metric in Eq. (14), $\alpha$ and $\delta$. For small $r$ near the Galactic core we adopt the Thomas-Fermi(TF) approximation for the energy density and the pressure of neutrinos, as in the case of fermion stars [9] 10 111 . From the local conservation law, $0=\left(\bar{\Psi} \gamma^{\mu} \Psi\right)_{; \mu}=$ $\frac{1}{\sqrt{-g}} \partial_{\mu}\left(\sqrt{-g} \bar{\Psi} \gamma^{\mu} \Psi\right)$, we have the conserved, total number of neutrinos,

$$
N=\int d^{3} x \sqrt{-g} \bar{\Psi} \gamma^{t} \Psi=\int d^{3} x \sqrt{-g} \Psi^{\dagger} \Psi e_{0}^{t} .
$$

The number density of neutrinos in the TF approximation is given by

$$
\left\langle\Psi^{\dagger} \Psi\right\rangle_{T F}=\frac{2}{(2 \pi)^{3}} \int d^{3} q n_{q}=\frac{q_{F}^{3}(r)}{3 \pi^{2}},
$$

where the Fermi distribution $n_{q}=\theta\left(q_{F}-q\right)$ with the fermi momentum $q_{F}$. From Eq. (86) and the timeindependent Dirac's equation, $i \partial_{t} \Psi_{n}=E_{n} \Psi_{n}$, we can put

$$
\begin{aligned}
& \left\langle\frac{i}{2 \delta \alpha}\left(\bar{\Psi}_{n} \gamma^{0} \partial_{t} \Psi_{n}-\partial_{t} \bar{\Psi}_{n} \gamma^{0} \Psi_{n}\right)\right\rangle_{T F} \\
& =\frac{2}{(2 \pi)^{3}} \int d^{3} q n_{q} E_{n}(q) e_{0}^{t} \equiv \rho(r),
\end{aligned}
$$

where $\rho$ is the energy density of neutrinos and $e^{t}{ }_{0}=\frac{1}{\delta \alpha}$. The stress tensor of neutrinos has the following diagonal elements in the vierbein basis:

$$
\begin{aligned}
\left\langle\frac{\alpha}{2 i}\left(\bar{\Psi}_{n} \gamma^{1} \partial_{r} \Psi_{n}-\partial_{r} \bar{\Psi}_{n} \gamma^{1} \Psi_{n}\right)\right\rangle_{T F} & =p_{1}, \\
\left\langle\frac{1}{2 i r}\left(\bar{\Psi}_{n} \gamma^{2} \partial_{\theta} \Psi_{n}-\partial_{\theta} \bar{\Psi}_{n} \gamma^{2} \Psi_{n}\right)\right\rangle_{T F} & =p_{2},
\end{aligned}
$$

and

$$
\left\langle\frac{1}{2 i r \sin \theta}\left(\bar{\Psi}_{n} \gamma^{3} \partial_{\phi} \Psi_{n}-\partial_{\phi} \bar{\Psi}_{n} \gamma^{3} \Psi_{n}\right)\right\rangle_{T F}=p_{3},
$$

respectively.

We assume, in the spherically symmetric spacetime we consider, that $p_{1}=p_{2}=p_{3} \equiv p(r)$. From Dirac's equation (36) and its Hermitian conjugate, we have the relation;

$$
\begin{aligned}
& \frac{i}{\delta \alpha}\left(\bar{\Psi}_{n} \gamma^{0} \partial_{t} \Psi_{n}-\partial_{t} \bar{\Psi}_{n} \gamma^{0} \Psi_{n}\right) \\
& +i \alpha\left(\bar{\Psi}_{n} \gamma^{1} \partial_{r} \Psi_{n}-\partial_{r} \bar{\Psi}_{n} \gamma^{1} \Psi_{n}\right) \\
& +\frac{i}{r}\left(\bar{\Psi}_{n} \gamma^{2} \partial_{\theta} \Psi_{n}-\partial_{\theta} \bar{\Psi}_{n} \gamma^{2} \Psi_{n}\right) \\
& +\frac{i}{r \sin \theta}\left(\bar{\Psi}_{n} \gamma^{3} \partial_{\phi} \Psi_{n}-\partial_{\phi} \bar{\Psi}_{n} \gamma^{3} \Psi_{n}\right)=2 m_{e f f}(r) \bar{\Psi}_{n} \Psi_{n}
\end{aligned}
$$

with $m_{\text {eff }}(r)=m_{n}-\frac{g_{y}}{v} F^{2}(r)$. Since the left hand side of above equation is same as $i \Sigma$ in Eq. (56), we have the following relation in the $\mathrm{TF}$ approximation.

$$
\frac{i}{2}\langle\Sigma\rangle_{T F} \equiv \rho-3 p=m_{\mathrm{eff}} \frac{v}{2 g_{y}} Q,
$$

where we have defined $\frac{v}{2 g_{y}} Q(r) \equiv\left\langle\bar{\Psi}_{n} \Psi_{n}\right\rangle_{T F}$. Thus in the TF approximation the Einstein equations (57)-(60) read

$$
\begin{aligned}
& \frac{1}{2 \delta^{2}}\left(\delta^{2} \alpha^{2}\right),_{r r}-\frac{\left(\delta^{2} \alpha^{2}\right),_{r}}{4 \delta^{2}} \frac{\delta^{2},_{r}}{\delta^{2}}+\frac{1}{r} \frac{\left(\delta^{2} \alpha^{2}\right),_{r}}{\delta^{2}} \\
& =\kappa\left[-V+\frac{1}{2} \rho+\frac{3}{2} p\right], \\
& -\frac{1}{2 \delta^{2}}\left(\delta^{2} \alpha^{2}\right){ }_{r r}+\frac{\left(\delta^{2} \alpha^{2}\right),_{r}}{4 \delta^{2}} \frac{\delta^{2},{ }_{r}}{\delta^{2}}-\frac{1}{r} \alpha^{2},_{r} \\
& =\kappa\left[\alpha^{2}\left(F{ }_{r}\right)^{2}+V+\frac{1}{2} \rho-\frac{1}{2} p\right], \\
& \frac{1}{r^{2}}\left(1-\alpha^{2}\right)-\frac{1}{r} \alpha^{2},{ }_{r}-\frac{\alpha^{2}}{2 r} \frac{\delta^{2},_{r}}{\delta^{2}} \\
& =\kappa\left[\frac{F^{2}}{r^{2}}+V+\frac{1}{2} \rho-\frac{1}{2} p\right],
\end{aligned}
$$

which are consistent with those in the case of fermion stars [9]. The scalar field equation (21) reads

$\alpha^{2} F,_{r r}+\frac{\alpha^{2}}{2} F,_{r}\left(\frac{\delta^{2},_{r}}{\delta^{2}}+2 \frac{\alpha^{2},_{r}}{\alpha^{2}}+\frac{4}{r}\right)-\frac{2 F}{r^{2}}-\frac{\partial V}{\partial F}+Q F=0$.

If we assume that there exist regular solutions of the metric components, scalar fields, and the energy density and pressure of neutrinos to above equations (92)-(95) for small $r$, take series expansions as

$$
\begin{aligned}
\alpha^{2} & =\sum_{n \geq 0} a_{n} r^{n}, \quad \delta^{2}=\sum_{n \geq 0} b_{n} r^{n} \\
F & =\sum_{n \geq 0} \eta_{n} r^{n}, \quad \rho=\sum_{n \geq 0} \rho_{n} r^{n}, \quad p=\sum_{n \geq 0} p_{n} r^{n},(96)
\end{aligned}
$$

and substitute these expansions into Eqs. (92)-(95), then we get the following values for the coefficients.

$$
\begin{aligned}
& a_{0}=1, a_{1}=0, a_{2}=-\kappa\left[\frac{1}{3} \rho_{0}+\frac{\lambda}{12} \eta^{4}+\frac{1}{2} \eta_{1}^{2}\right], \\
& b_{1}=0, \quad b_{2}=b_{0} \kappa\left[\frac{1}{2} \rho_{0}+\frac{1}{2} p_{0}+\frac{1}{2} \eta_{1}^{2}\right], \\
& \eta_{0}=0, \quad \eta_{2}=0, \quad m_{n} \frac{v}{2 g_{y}} Q_{0}=\rho_{0}-3 p_{0} \ldots
\end{aligned}
$$

We thus have asymptotic solutions as

$$
\begin{aligned}
F & =\eta_{1} r+\mathcal{O}\left(r^{3}\right) \\
\alpha^{2} & =1-\kappa\left[\frac{1}{3} \rho_{0}+\frac{\lambda}{12} \eta^{4}+\frac{1}{2} \eta_{1}^{2}\right] r^{2}+\mathcal{O}\left(r^{3}\right), \\
\delta^{2} \alpha^{2} & =\left\{1+\kappa\left[\frac{1}{6} \rho_{0}+\frac{1}{2} p_{0}-\frac{\lambda}{12} \eta^{4}\right] r^{2}\right\}+\mathcal{O}\left(r^{3}\right),
\end{aligned}
$$

where we have reparametrized the time as $\sqrt{b_{0}} t \rightarrow$ $t$. These solutions are consistent with the results in fermionic stars with a global monopole 11, even if they 
111 take $F \simeq 0$ for small $r$. The value of $\eta_{1}$ is determined by the matching condition that $F(r)$ should be continuous at $r=r_{c}$, which is the upper bound for small $r$. This gives us the relation,

$$
\eta_{1} r_{c}=\eta
$$

We might put $r_{c} \simeq 2 M_{S B H}$ where $M_{S B H}$ is the mass of the supermassive black hole at the center of our Galaxy [12].

\section{NEUTRINO CLUSTERING}

In Z-burst models to explain the continuation of the cosmic ray spectrum beyond the GZK cutoff, Weiler [6] and Fargion [13] assumed that the relic neutrinos cluster in galaxies a few times the normal relic density, and found that the required flux of cosmic ray neutrinos is larger than previously suggested. Blanco-Pillado et al. 14. suggested that the relic neutrino density in the clustering might be $10^{12}-10^{14}$ times the mean relic density, $n_{M} \simeq$ $54 \mathrm{~cm}^{-3}$. Independently in Ref. [5] a neutrino cloud was considered as a sphere with no diffuse boundary such that the relic neutrino density is given by $n_{\nu}(r)=n_{R} \theta(R-$ $r)+n_{M} \theta(r-R)$, where $n_{R} \simeq 10^{12}-10^{16} \mathrm{~cm}^{-3}$ and $R \simeq 10^{14}-10^{20} \mathrm{~cm}$. This can moderate the required incident flux of ultra high energy neutrinos.

From the results in the previous sections IV and V, we can have a cluster of neutrinos with a diffuse boundary such that the density of the relic neutrinos is

$$
n_{\nu}(r)=n_{c} \theta\left(r_{c}-r\right)+n_{o}(r) \theta\left(r-r_{c}\right) \theta\left(r_{o}-r\right),
$$

where $n_{c}$ is the constant neutrino density for small $r(\leq$ $\left.r_{c} \sim 2 M_{S B H}\right)$ as in the section $\mathrm{V}$, and $n_{o}(r)=n_{c} \frac{r_{c}^{2}}{r^{2}}$ is the neutrino density for $r_{c}<r \leq r_{o}$ as in the section IV. Here $r_{o} \simeq 10^{23} \mathrm{~cm}$ (; the radius of our Galaxy), $r_{c} \simeq 2 M_{S B H} \simeq 10^{12} \mathrm{~cm}$ (; the Schwarzschild radius of a supermassive black hole at the center of the Galaxy), and $n_{c} \geq 10^{3}\left(\frac{m_{e f f}}{1 e V}\right)^{3} \mathrm{~cm}^{-3}$ 15.

\section{SUMMARY AND DISCUSSIONS}

In the most general static spacetime with spherical symmetry, we explicitly show that only $j=1 / 2$ fermions can satisfy Einstein's equation, in the $\vec{\rho} \otimes \vec{\sigma}$ representation of $\gamma$-matrices $[7$. It is also possible to show the fact in other representations of $\gamma$-matrices. Considering neutrinos coupled to $O(3)$ scalar fields $\vec{\Phi}$ via $\vec{\Phi}^{2} \bar{\Psi} \Psi$ interaction term, we had a global monopole solution [1] of scalar fields and asymptotic solutions of the metric components to Einstein's equation in Eqs. (78) and (80), for large $r$. The asymptotic solution of one metric component $\delta^{2}$ can be given in series of more general functions as $\delta^{2}(r) \simeq \sum_{n} f_{n}{ }^{2}(r) \ln ^{n}\left(\frac{r}{2 M}\right)$ with $f_{n}(r \rightarrow \infty)=$ constant, which do not change the asymptotic behaviors of other solutions in this limit.

We adopted the Thomas-Fermi(TF) approximation for small $r$ expansions of the energy density, pressure of neutrinos and so on. Since only $s$-waves are considered in the TF approximation, small $r$ expansions in the section $\mathrm{V}$ are consistently related to asymptotic solutions obtained for large $r$ in th esection IV. More rigorous connection between two asymptotic solutions can be made by numerical methods. With the simple assumption, Eq. (100), about the radial dependence of the relic density of neutrinos clustered in the galactic halo, further studies of Z-burst models shall be possible. For small $r$ solutions in the section $\mathrm{V}$ to have astronomical meaning, the global $O(3)$ symmetry breaking scale $\eta$ and the scalar self-coupling constant $\lambda$ in Eq. (12) should be very small, which might be realized in some Majoron models [17]. In such a case the deficit angle $1-c \equiv \kappa \eta^{2}$ in Eq. (78) is negligible [1].

\section{ACKNOWLEDGEMENTS}

THL would like to thank School of Physics, The University of Melbourne for the hospitality extended to him during the completion of this article. This work was supported by Korea Research Foundation Grant(KRF-2001015-DP0091).
[1] M. Barriola and A. Vilenkin, Phys. Rev. Lett. 63, 341 (1989).

[2] U. Nucamendi, M. Salgado and D. Sudarsky, Phys. Rev. D 63, 125016 (2001); U. Nucamendi, M. Salgado and D. Sudarsky, Phys. Rev. Lett. 84, 3037 (2000).

[3] D. Harari and C. Loustó, Phys. Rev. D 42, 2626 (1990).

[4] L. A. Boyle, R. R. Caldwell, and M. Kamionkowski, astro-ph/0105318; S. Kasuya, astro-ph/0105408; X. Li,
J. Hao, and D. Liu, astro-ph/0107171; B. J. Lee and T. H. Lee, Int. Jr. of Mod. Phys. A 17, 555 (2002).

[5] G. J. Stephenson Jr., T. Goldman and B. H. J. McKellar, Int. J. Mod. Phys. A 13, 2765, (1998); B. H. J. McKellar, M. Garbutt, G. J. Stephenson Jr. and T. Goldman, hep$\mathrm{ph} / 0106123$.

[6] T. J. Weiler, Phys. Rev. Lett. 49, 234 (1982); T. J. Weiler, Astropart. Phys. 11, 303 (1999); hep- 
ph/9710431.

[7] P. A. M. Dirac, in The Principle of Quantum Mechanics (Oxford Univ. Press, London 1958).

[8] D. G. Boulware, Phys. Rev. D 12, 350 (1975).

[9] T. D. Lee and Y. Pang, Phys. Rev. D 35, 3678 (1987).

[10] S. Bachall, B. W. Lynn and S. B. Selipsky, Nucl. Phys. B 325, 606 (1989).

[11] X. Z. Li and X. H. Zhai, Phys. Lett. B 364, 212 (1995).

[12] A. M. Ghez, B. L. Klein, M. Morris and E. E. Becklin, astro-ph/9807210; A. Goldwurm, astro-ph/0102382.

[13] D. Fargion, B. Mele and A, Salis, Astophys. J. 517, 725
(1999).

[14] J. J. Blanco-Pillado, R. A. Vázquez, and E. Zas, Phys. Rev. D 61, 123003, 2000.

[15] E. Waxman, astro-ph/9804023.

[16] J. D. Anderson, E. L. Lau, T. P. Krisher, D. A. Dicus, D. C. Rosenbaum and V. L. Teplitz, Astrophys. J. 448, 885 (1995).

[17] X. G. He, B. H. J. Mckellar and G. J. Stephenson Jr., hep-ph/9807338 E. Ma, M. Raidal and U. Sarkar, hepph/0012101. 